\title{
Measurement of the Boron to Carbon Flux Ratio in Cosmic Rays with the DAMPE Experiment
}

\author{
Chuan Yue, ${ }^{a, *}$ Zhan-Fang Chen, ${ }^{a, b}$ Ming-Yang Cui, ${ }^{a}$ Dimitrios Kyratzis ${ }^{c, d}$ and Li-Bo \\ $\mathrm{Wu}^{c, d, e}$ on behalf of the DAMPE Collaboration \\ (a complete list of authors can be found at the end of the proceedings)
}

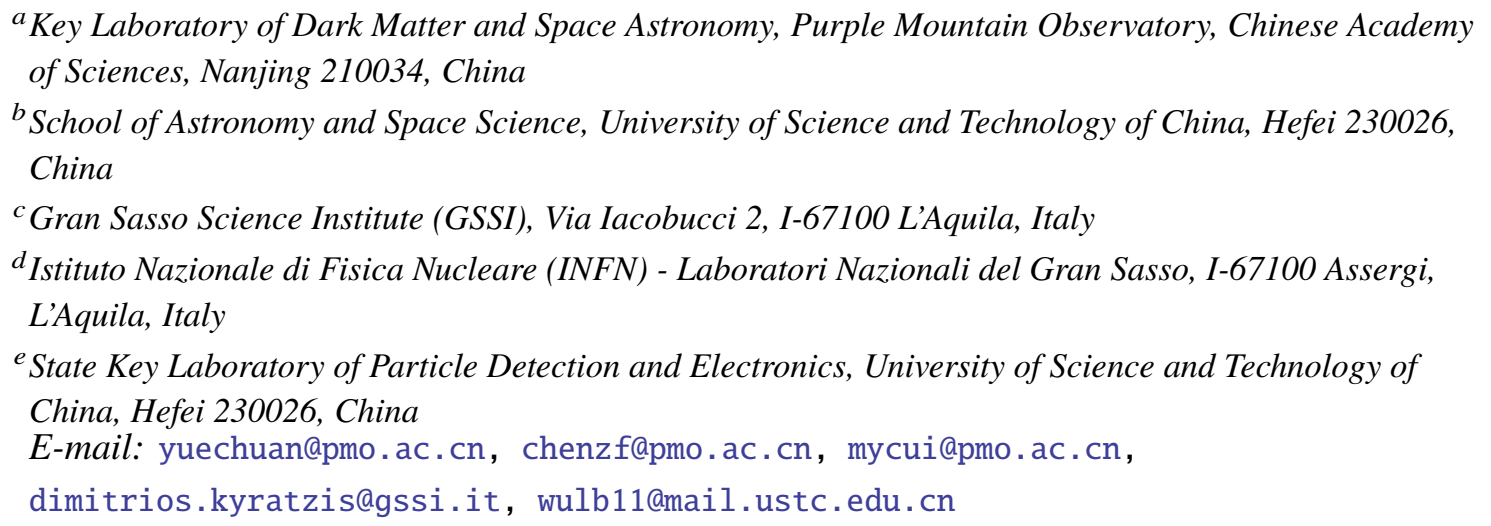

The DArk Matter Particle Explorer (DAMPE), a space-based high energy particle detector, has been operated on-orbit for more than five years. The large geometric factor and good charge resolution enable DAMPE to have very good potential to measure cosmic-rays up to $100 \mathrm{TeV}$. Knowledge of the boron to carbon $(\mathrm{B} / \mathrm{C})$ flux ratio is very important in understanding the propagation of cosmic rays, especially in $\mathrm{TeV}$ energy range. In this contribution, the latest progress of the B/C flux ratio analysis based on the flight data collected by DAMPE during the 5 years of operation, is presented.

$37^{\text {th }}$ International Cosmic Ray Conference (ICRC 2021)

July 12 th - 23rd, 2021

Online - Berlin, Germany

\footnotetext{
*Presenter
} 


\section{Introduction}

Cosmic rays (CRs) are conventionally divided into two classes: primary and secondary. Primary CRs are those particles accelerated at astrophysical sources, and secondaries are those particles produced in interaction of the primaries with interstellar gas. Recent observations from ASM-02 indicate a identical spectral hardening at $\sim 200 \mathrm{GV}$ for secondary cosmic rays $(\mathrm{Li}, \mathrm{Be}$, and $\mathrm{B})$ and primary cosmic rays ( $\mathrm{He}, \mathrm{C}$ and $\mathrm{O}$ ), but the rigidity dependences of these two groups are distinctly different $[1,2]$ In particular, the boron nuclei are thought to be entirely produced by the collision of heavier nuclei, such as carbon and oxygen, with nuclei of the interstellar matter. Therefore, the boron to carbon flux ratio directly indicates the average amount of interstellar material traversed by cosmic rays [3].

Because of the importance of the $\mathrm{B} / \mathrm{C}$ flux ratio to the understanding of cosmic rays, over the last 20 years there have been many measurements [4-7]. Precise measurement of the B/C flux Ratio by AMS-02 [7], shows that the B/C ratio is well described by a single power law $R^{\Delta}$ with index $\Delta=-0.333$ above $65 \mathrm{GV}$ rigidities, in good agreement with the Kolmogorov theory of turbulence which predicts $\Delta=-1 / 3$ asymptotically [8]. In TV rigidities, However, the current measurements from the magnetic spectrometers have very large errors and cannot clarify if the $\mathrm{B} / \mathrm{C}$ flux ratio remains a single power law in high rigidities.

The DArk Matter Particle Explorer (DAMPE) is a calorimetric-type, satellite-borne detector for high energy electron/positron, $\gamma$-ray, and CR observations[9]. Since launched on Dec.17th 2015, DAMPE has obtained advanced measurements of $\mathrm{CR} e \pm$ spectrum[10], proton spectrum [11], and helium spectrum[12], and revealed new spectral structures with high confidences. With a large geometric factor and a good charge resolution[13], DAMPE is expected to extend the measurement of the $\mathrm{B} / \mathrm{C}$ flux ratio up to a few $\mathrm{TeV} / \mathrm{n}$ energies.

\section{DAMPE instrument}

The DAMPE detector consists of 4 sub-detectors, which are a Plastic Scintillator strip Detector (PSD)[? ], a Silicon-Tungsten tracKer-converter(STK)[16], a BGO imaging calorimeter [17] and a NeUtron Detector (NUD) [18] from top to bottom. The PSD measures the charge of an incident particle It can also be used as an anti-coincidence detector for $\gamma$-rays. The STK reconstructs the trajectory and measures the charge. The BGO calorimeter measures the energy, and provides electron-hadron identification. The track information can also be obtained via the BGO image. The NUD provides additional electron-hadron discrimination, which is important for energies above $\mathrm{TeV}$. These 4 sub-detectors enable good measurements of the charge $(|Z|)$, arrival direction, energy, and particle identity of each event.[9]

\section{Data analysis}

Five years of DAMPE on-orbit data from January $1^{s t}$, 2016 to December $31^{s t}, 2020$ are analyzed in this work. The total live time is $1.1977446 \times 10^{8} \mathrm{~s}$ after excluding the time when the satellite passes the South Atlantic Anomaly (SAA) region, the instrument dead time, the time for on-orbit calibration, and the period between September 9, 2017 and September 13, 2017 when a big solar flare occurred and may have affected the baseline of the detector. 


\subsection{Event Selection}

To ensure that all detectors work in good condition, data recorded when the satellite was in the SAA region are excluded. Furthermore, we select events with deposited energy in the calorimeter larger than $100 \mathrm{GeV}$, to effectively avoid the effect due to the geomagnetic rigidity cutoff [14] for boron and carbon nuclei.

\section{- Pre-selection}

The DAMPE has four different triggers implemented on orbit [9]: Unbiased trigger, MIP trigger, High-Energy (HE) trigger and Low-Energy(LE) trigger. The events are required to meet the HE trigger condition in order to make sure the shower development starts at the beginning of the calorimeter. For boron and carbon nuclei, with deposited energy larger $100 \mathrm{GeV}$, the $\mathrm{HE}$ trigger efficiency is as high as $100 \%$. Apart from the HE trigger, the pre-selection requires at least one hit in each sub-layer of the PSD and at least one good track in the STK with more than four hits in both $X$ and $Y$ views. In addition, the good track should transverse the PSD hit with maximum energy in both $X$ and $Y$ views, the reduced $\chi^{2}$ value should be smaller than 5. In case that there are more than one good tracks in the STK, the most reliable one is selected by jointly considering the following factors: the length of the track, the reduced $\chi^{2}$ value of the fit, and the match between the candidate track and the shower axis in the calorimeter. The selected track is then required to cross all sub-layers of the PSD and pass through the calorimeter from top to bottom.
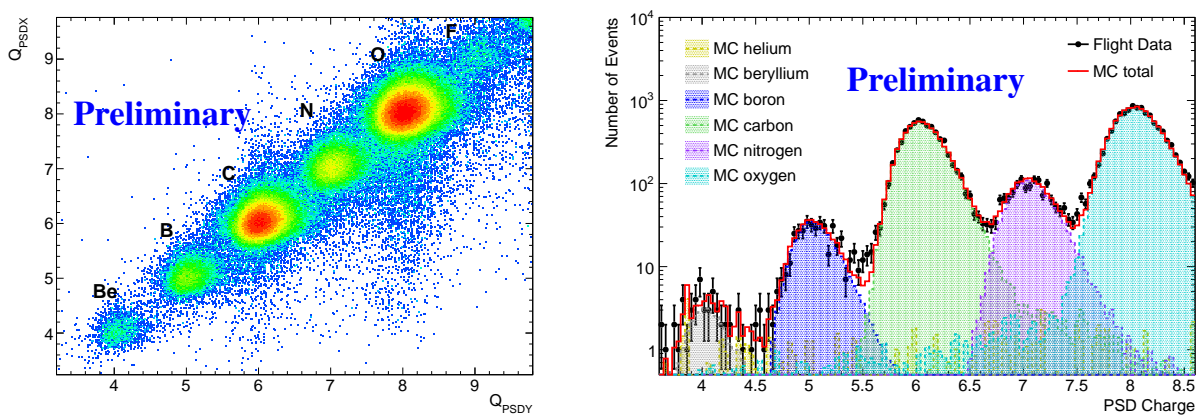

Figure 1: Left - PSDY versus PSDX reconstructed charges in the elemental range between Be and F. Right - The combined PSD charge template fitting for deposited energies of $1000-1412 \mathrm{GeV}$ in the BGO calorimeter.

\section{- Charge selection}

Firstly, we require that the signal of the first hit on the STK track should be larger than 20 times of the MIP-equivalent signal, and the charge RMS of all hits on the STK track should be smaller than 2. Then, the charge measurements of PSD are used to select boron and carbon candidates. The PSD is composed of two layers placed in a hodoscopic configuration ( $Y$-view for layer-1 and $X$-view for layer-2), with 41 plastic scintillator strips in each layer [? ]. The PSD charge reconstruction procedure includes light attenuation (position) correction, light yield saturation (quenching) correction, energy independence. For an incident charge 
particle, the charge is measured independently by the two PSD layers. Figure 1 left shows the crossplot of PSDY versus PSDX reconstructed charges in the elemental range between $\mathrm{Be}$ and $\mathrm{F}$.

The charge measurements of the MC simulations show an energy-dependent difference from that of the flight data primarily due to the back-scattering secondaries. To mitigate such an effect, an energy-dependent charge correction is applied for the MC data. We first parameterize the charge distributions of protons and helium nuclei in different deposited energy bins with a Landau-Gaussian convolution function for the flight data and MC data separately. Then the MC charges are shifted and shrinked according to the best-fitting parameters to match with the flight data.

The combined PSD charge distribution after corrections for deposited energies of 1000-1425 $\mathrm{GeV}$ is plotted in the right panel of Figure 1, where the boron and carbon peaks can be clearly seen. Two constant cut ranges, [4.75,5.35] and [5.7,6.5], are applied to select boron and carbon candidates respectively. The charge selection efficiencies for boron and carbon are validated by the comparison of flight data and MC data. The deviation of the flight data from the $\mathrm{MC}$ simulations is within $2 \%$ for boron and $1 \%$ for carbon.
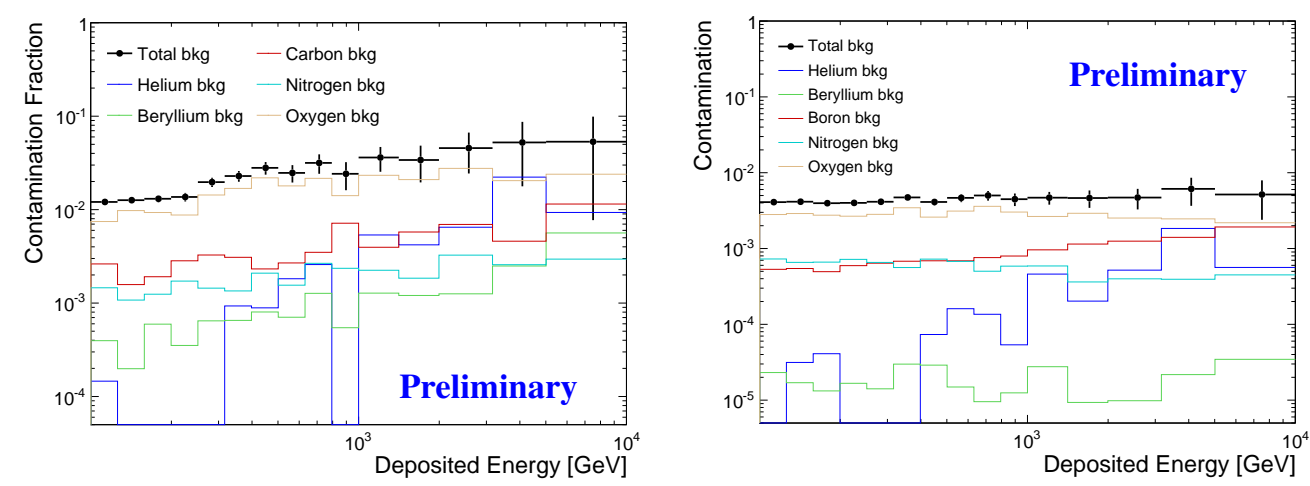

Figure 2: Left — The contamination fractions for boron. Right - The contamination fractions for carbon.

\subsection{Background}

The background for boron or carbon includes different mis-identified nuclear species. The contamination from proton and nuclei heavier than fluorine is negligible for both boron and carbon. Background contamination fractions from different nuclear species misidentified as boron or carbon is shown in Figure 2. For boron, the total contamination fraction is $\lesssim 2 \%$ below $300 \mathrm{GeV}$, and increases up to $\sim 5 \%$ around $10 \mathrm{TeV}$, dominated by helium nuclei. For carbon, the total contamination fraction remains $\lesssim 1 \%$ below $10 \mathrm{TeV}$.

\subsection{Effective Acceptance}

The effective acceptance is defined as the product of the geometric factor and selection efficiencies (including trigger, track, and charge selections). To estimate the effective acceptance, a 
detailed MC simulation using the GEANT4 toolkit [19] is performed. A digitization algorithm including the DAMPE detector response is developed for converting the raw energy hits by GEANT4 simulations into Analog-to-Digital Converter (ADC) counts. Then we apply the reconstruction algorithm to the simulation data to obtain the reconstructed events. An isotropic spectrum with $E^{-1.0}$ power-law is generated for detector simulation using the GEANT FTFP_BERT physicslist [20], then is re-weighted to $E^{-3.0}$ for boron and $E^{-2.7}$ for carbon. The effective acceptance of the $i$-th incident energy bin is obtained by

$$
A_{\mathrm{eff}, i}=A_{\mathrm{gen}} \times \frac{N_{\mathrm{pass}, i}}{N_{\mathrm{gen}, i}}
$$

where $A_{\mathrm{gen}}$ is the geometrical factor of the MC generation sphere, $N_{\mathrm{gen}, i}$ and $N_{\mathrm{pass}, i}$ are the numbers of generated events and those passing the selections.

\subsection{Energy Unfolding}

Due to the limited thickness of the BGO calorimeter ( 1.62 nuclear length), a boron or carbon particle does not fully deposit its energy in the calorimeter. To convert the measured energy spectrum to the primary energy spectrum, it is necessary to unfold the instrument response. Instead of correcting the particle energy event-by-event, the unfolding procedure enables an estimate of the energy distribution of incident particles from the deposit energy distribution.

The number of events in the $i$-th deposited energy bin, $N_{\text {dep }, i}$, can be obtained via the sum of number of events $N_{\mathrm{inc}, j}$ in all the incident energy bins weighted by the energy response matrix

$$
N_{\text {dep }, i}=\sum_{j} M_{i j} N_{\text {inc }, j}
$$

where $M_{i j}$, the energy response matrix, is the probability that an event in the $j$-th incident energy bin is detected in the $i$-th deposited energy bin. Eq. 2 is solved with a Bayesian method to derive the incident event distribution[21].

\subsection{Uncertainties}

The statistical uncertainties refer to the Possion fluctuations of the detected numbers of events in each deposited energy bin. To get the statistical uncertainties of the unfolded proton fluxes, an error propagation from the detected events to the unfolded fluxes is necessary in order to properly take into account the bin-by-bin migration due to the unfolding procedure[21].

The systematic uncertainties related with the charge selection are estimated through comparisons between flight data and MC simulations. For the STK charge selection, the efficiency differences are $[-2 \%, 3.16 \%]$ and $[-1 \%, 1 \%]$ for boron and carbon, corresponding to an uncertainty of $[-2.24 \%, 3.32 \%]$ for $\mathrm{B} / \mathrm{C}$ flux ratio. For the PSD charge selection, the efficiency differences are $[-2 \%, 2 \%]$ and $[-1 \%, 1 \%]$ for boron and carbon, corresponding to an uncertainty of $[-2.24 \%$, $2.24 \%$ ] for $\mathrm{B} / \mathrm{C}$ flux ratio.

The uncertainties due to the spectral deconvolution are estimated to be $\lesssim 1 \%$, through regeneration of the response matrix and varying the spectral index from 2.5 to 3.2 when re-weighting the simulation data. The systematic uncertainties due to the background subtraction are estimated through varying the charge selection condition from $-10 \%$ to $5 \%$ for both of boron and carbon, and 


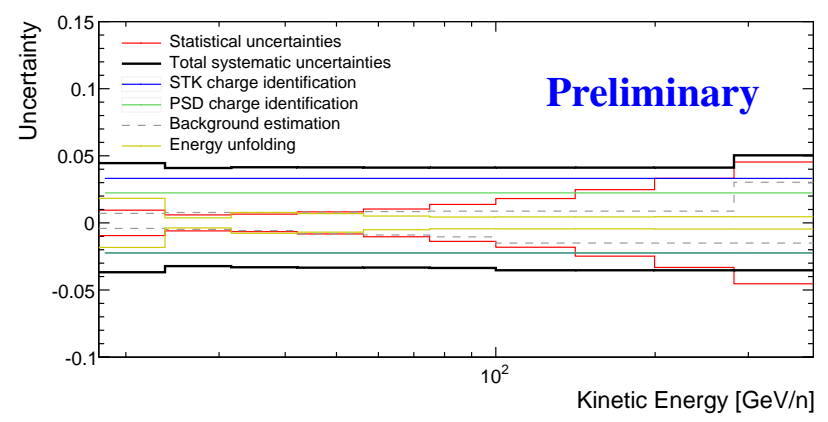

Figure 3: Energy (per nucleon in $\mathrm{GeV}$ ) dependence of systematic uncertainties (relative errors) for $\mathrm{B} / \mathrm{C}$ flux ratio.

repeating the analysis. The background subtraction gives $\sim 1 \%$ systematic uncertainties below 100 $\mathrm{GeV} / \mathrm{n}$ and increases to $\sim 2.5 \%$ at $400 \mathrm{GeV} / \mathrm{n}$.

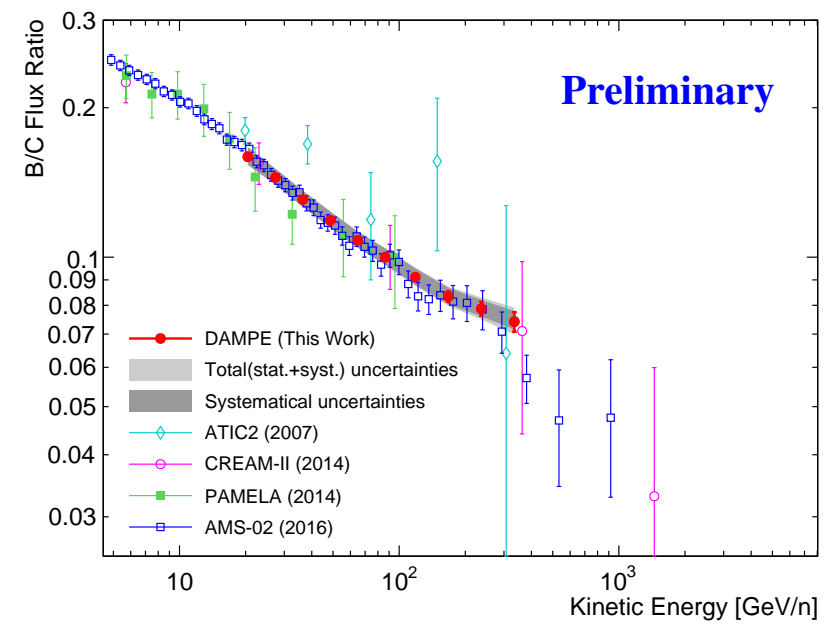

Figure 4: B/C flux ratio from $15 \mathrm{GeV} / \mathrm{n}$ to $3 \mathrm{TeV} / \mathrm{n}$ measured with DAMPE (red points), compared with previous results by ATIC-2 [4], CREAM-II [5], PAMELA [6], AMS-02 [7]. The red error bars show the statistical uncertainties, the gray profiles show the quadratic sums of statistical and systematic uncertainties.

\section{Results}

The $\mathrm{B} / \mathrm{C}$ flux ratio in the energy range from $15 \mathrm{GeV} / \mathrm{n}$ to $3 \mathrm{TeV} / \mathrm{n}$ is shown in Figure 4 . The error bars represent the statistical uncertainties, the gray profiles show the quadratic sums of statistical and systematic uncertainties. Previous measurements by space detectors ATIC-2 [4], CREAM-II [5], PAMELA [6] and AMS-02 [7] are overlaid for comparison. The DAMPE result is well consistent with those of PAMELA and AMS-02 within uncertainties.

More detailed analysis of the DAMPE data and more precise estimation of systematic uncertainties are on-going. With the accumulation of data, DAMPE is expected to measure the B/C flux ratio up to a few $\mathrm{TeV} / \mathrm{n}$ with good precision, which will represent a significant step forward in understanding the propogation of CRs. 


\section{Acknowledge}

The DAMPE mission is funded by the strategic priority science and technology projects in space science of Chinese Academy of Sciences. In China the data analysis is supported by the National Key Research and Development Program of China (No. 2016YFA0400200), the National Natural Science Foundation of China (Nos. 11525313, 11622327, 11722328, 11851305, 11921003, 12003076, U1738205, U1738206, U1738207, U1738208, U1738127), the strategic priority science and technology projects of Chinese Academy of Sciences (No. XDA15051100), the Young Elite Scientists Sponsorship Program by CAST (No. YESS20160196), and the Program for Innovative Talents and Entrepreneur in Jiangsu. In Europe the activities and data analysis are supported by the Swiss National Science Foundation (SNSF), Switzerland, the National Institute for Nuclear Physics (INFN), Italy, and the European Research Council (ERC) under the European Union's Horizon 2020 research and innovation programme (No. 851103).

\section{References}

[1] M. Aguilar et al. [AMS Collaboration], Observation of the Identical Rigidity Dependence of $\mathrm{He}, \mathrm{C}$, and $\mathrm{O}$ Cosmic Rays at High Rigidities by the Alpha Magnetic Spectrometer on the International Space Station, Phys. Rev. Lett. 119 (2017) 251101.

[2] M. Aguilar et al. [AMS Collaboration], Observation of New Properties of Secondary Cosmic Rays Lithium, Beryllium, and Boron by the Alpha Magnetic Spectrometer on the International Space Station, Phys. Rev. Lett. 120 (2018) 021101.

[3] A. W. Strong, I. V. Moskalenko, and V.S. Ptuskin, Cosmic-ray propagation and interactions in the Galaxy Annu. Rev. Nucl. Part. Sci. 57 (2007) 285.

[4] A. D. Panov, et al., Proceedings of the 30th International Cosmic Ray Conference 2 (2008) 3. arXiv: 0707.4415

[5] H. S. Ahn, et al., Astropart. Phys. 30 (2008) 133.

[6] O. Adriani,et al., Astrophys. J. 791 (2014) 93.

[7] M. Aguilar et al. [AMS Collaboration], Precision Measurement of the Boron to Carbon Flux Ratio in Cosmic Rays from 1.9 GV to 2.6 TV with the Alpha Magnetic Spectrometer on the International Space Station, Phys. Rev. Lett. 117 (2016) 231102.

[8] A. N. Kolmogorov, Proc. R. Soc. 434 (1991) 9-13.

[9] J. Chang et al. [DAMPE Collaboration], The Dark Matter Particle Explorer mission, Astropart. Phys. 95 (2017) 6-24.

[10] G. Ambrosi et al. [DAMPE collaboration] Direct detection of a break in the teraelectronvolt cosmic-ray spectrum of electrons and positrons Nature, 552 (2017) 63.

[11] Q. An et al. [DAMPE collaboration] Measurement of the cosmic ray proton spectrum from $40 \mathrm{GeV}$ to $100 \mathrm{TeV}$ with the DAMPE satellite Sci. Adv. , 5 (2019) eaax3793. 
[12] F. Alemanno et al. [DAMPE collaboration] Measurement of the Cosmic Ray Helium Energy Spectrum from $70 \mathrm{GeV}$ to $80 \mathrm{TeV}$ with the DAMPE Space Mission Phys. Rev. Lett. 126 (2020) 201102.

[13] T.-K. Dong, Y.-P. Zhang, P.-X. Ma, et al., Charge measurement of cosmic ray nuclei with the plastic scintillator detector of DAMPE. Astropart. Phys. , 105(2019) 31-36.

[14] E. Thébault et al., International Geomagnetic Reference Field: the 12th generation, Earth, Planets and Space 67 (2017) 79.

[15] Y.-H. Yu et al., The Plastic Scintillator Detector at DAMPE, Astropart. Phys. 94 (2017) 1-10.

[16] P. Azzarello et al., The DAMPE silicon-tungsten tracker, Nucl. Instrum. Meth. A 831 (2017) 379.

[17] Z.-Y. Zhang et al., The calibration and electron energy reconstruction of the BGO ECAL of the DAMPE detector, Nucl. Instrum. Meth. A 836 (2016), 98.

[18] Y.-Y. Huang, T. Ma, C. Yue et al., Calibration and performance of the neutron detector onboard of the DAMPE mission, Res. Astron. Astrophys. 20 (2021) 153.

[19] S. Agostinelli, et al., Geant4 - a simulation toolkit Nucl. Instrum. Meth. A 506 (2017) 3.

[20] Geant4 version employs FTFP_BERT as a recommended physics list for the simulation of high energy hadronic showers. http://geant4-userdoc.web.cern.ch/geant4userdoc/UsersGuides/PhysicsListGuide/html/physicslistguide.html

[21] G. D'Agostini, A multidimensional unfolding method based on Bayes' theorem, Nuclear Instruments and Methods in Physics Research A 362 (1995) 362. 


\section{Full Authors List: DAMPE Collaboration}

F. Alemanno ${ }^{1,2}$, Q. An ${ }^{3,4}$, P. Azzarello ${ }^{5}$, F. C. T. Barbato ${ }^{1,2}$, P. Bernardini ${ }^{6,7}$, X. J. Bi ${ }^{8,9}$, M. S. Cai ${ }^{10,11}$, E. Casilli6, ${ }^{6,7}$, E. Catanzani ${ }^{12}$, J. Chang ${ }^{10,11}$, D. Y. Chen ${ }^{10}$, J. L. Chen ${ }^{13}$, Z. F. Chen ${ }^{10,11}$, M. Y. Cui ${ }^{10}$, T. S. Cui ${ }^{14}$, Y. X. Cui ${ }^{10,11}$, H. T. Dai ${ }^{3,4}$, A. De Benedittis ${ }^{6,7}$, I. De Mitri ${ }^{1,2}$, F. de Palma ${ }^{6,7}$, M. Deliyergiyev ${ }^{5}$, M. Di Santo ${ }^{1,2}$, Q. Ding ${ }^{10,11}$, T. K. Dong ${ }^{10}$, Z. X. Dong ${ }^{14}$, G. Donvito ${ }^{15}$, D. Droz ${ }^{5}$, J. L. Duan ${ }^{13}$, K. K. Duan ${ }^{10}$, D. D’Urso ${ }^{12, *}$, R. R. Fan ${ }^{8}$, Y. Z. Fan ${ }^{10,11}$, K. Fang ${ }^{8}$, F. Fang ${ }^{13}$, C. Q. Feng ${ }^{3,4}$, L. Feng ${ }^{10}$, P. Fusco ${ }^{15,16}$, M.

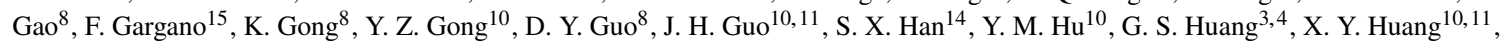
Y. Y. Huang ${ }^{10}$, M. Ionica ${ }^{12}$, W. Jiang ${ }^{10}$, J. Kong ${ }^{13}$, A. Kotenko ${ }^{5}$, D. Kyratzis ${ }^{1,2}$, S. J. Lei ${ }^{10}$, W. H. Li ${ }^{10,11}$, W. L. Li ${ }^{14}$, X. Li ${ }^{10}$, X. Q.

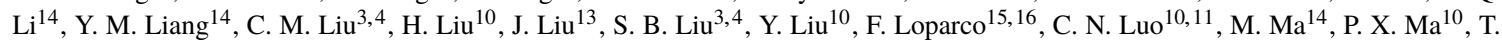

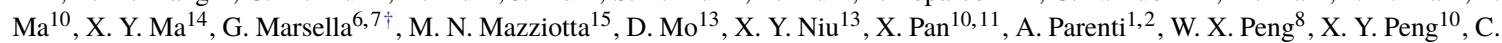
Perrina $^{5,}$, R. Qiao $^{8}$, J. N. Rao ${ }^{14}$, A. Ruina ${ }^{5}$, M. M. Salinas ${ }^{5}$, G. Z. Shang ${ }^{14}$, W. H. Shen ${ }^{14}$, Z. Q. Shen ${ }^{10}$, Z. T. Shen ${ }^{3,4}$, L. Silveri ${ }^{1,2}$, J. X. Song ${ }^{14}$, M. Stolpovskiy ${ }^{5}$, H. Su ${ }^{13}$, M. Su ${ }^{17}$, H. R. Sun ${ }^{3,4}$, Z. Y. Sun ${ }^{13}$, A. Surdo ${ }^{7}$, X. J. Teng ${ }^{14}$, A. Tykhonov ${ }^{5}$, H. Wang ${ }^{14}$, J. Z.

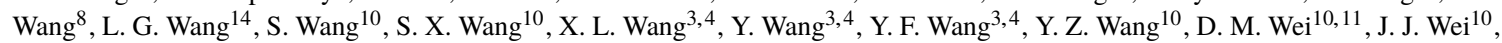
Y. F. Wei ${ }^{3,4}$, D. $\mathrm{Wu}^{8}$, J. Wu ${ }^{10,11}$, L. B. Wu ${ }^{1,2}$, S. S. $\mathrm{Wu}^{14}$, X. Wu $\mathrm{Wu}^{5}$ Z. Q. Xia ${ }^{10}$, H. T. Xu ${ }^{14}$, Z. H. Xu ${ }^{10,11}$, Z. L. Xu ${ }^{10}$, E. H. Xu ${ }^{3,4}, Z$. Z. Xu ${ }^{3,4}$, G. F. Xue ${ }^{14}$, H. B. Yang ${ }^{13}$, P. Yang ${ }^{13}$, Y. Q. Yang ${ }^{13}$, H. J. Yao ${ }^{13}$, Y. H. Yu ${ }^{13}$, G. W. Yuan ${ }^{10,11}$, Q. Yuan ${ }^{10,11}$, C. Yue ${ }^{10}$, J. J. Zang $^{10}$ S. X. Zhang ${ }^{13}$, W. Z. Zhang ${ }^{14}$, Yan Zhang ${ }^{10}$, Yi Zhang ${ }^{10,11}$, Y. J. Zhang ${ }^{13}$, Y. L. Zhang ${ }^{3,4}$, Y. P. Zhang ${ }^{13}$, Y. Q. Zhang ${ }^{10}$, Z. Zhang $^{10}$, Z. Y. Zhang ${ }^{3,4}$, C. Zhao ${ }^{3,4}$, H. Y. Zhao ${ }^{13}$, X. F. Zhao ${ }^{14}$, C. Y. Zhou ${ }^{14}$, and Y. Zhu ${ }^{14}$

${ }^{1}$ Gran Sasso Science Institute (GSSI), Via Iacobucci 2, I-67100 L'Aquila, Italy

${ }^{2}$ Istituto Nazionale di Fisica Nucleare (INFN) -Laboratori Nazionali del Gran Sasso, I-67100 Assergi, L'Aquila, Italy

${ }^{3}$ State Key Laboratory of Particle Detection and Electronics, University of Science and Technology of China, Hefei 230026, China

${ }^{4}$ Department of Modern Physics, University of Science and Technology of China, Hefei 230026, China

${ }^{5}$ Department of Nuclear and Particle Physics, University of Geneva, CH-1211, Switzerland

${ }^{6}$ Dipartimento di Matematica e Fisica E. De Giorgi, Universita‘ del Salento, I-73100, Lecce, Italy

${ }^{7}$ Istituto Nazionale di Fisica Nucleare (INFN) - Sezione di Lecce, I-73100, Lecce, Italy

${ }^{8}$ Institute of High Energy Physics, Chinese Academy of Sciences, Yuquan Road 19B, Beijing 100049, China

${ }^{9}$ University of Chinese Academy of Sciences, Yuquan Road 19A, Beijing 100049, China

${ }^{10}$ Key Laboratory of Dark Matter and Space Astronomy, Purple Mountain Observatory, Chinese Academy of Sciences, Nanjing 210023 , China

${ }^{11}$ School of Astronomy and Space Science, University of Science and Technology of China, Hefei 230026, China

${ }^{12}$ Istituto Nazionale di Fisica Nucleare (INFN) - Sezione di Perugia, I-06123 Perugia, Italy

${ }^{13}$ Institute of Modern Physics, Chinese Academy of Sciences, Nanchang Road 509, Lanzhou 730000, China

${ }^{14}$ National Space Science Center, Chinese Academy of Sciences, Nanertiao 1, Zhongguancun, Haidian district, Beijing 100190, China

${ }^{15}$ Istituto Nazionale di Fisica Nucleare (INFN) - Sezione di Bari, I-70125, Bari, Italy

${ }^{16}$ Dipartimento di Fisica “M. Merlin" dell'Universita' e del Politecnico di Bari, I-70126, Bari, Italy

${ }^{17}$ Department of Physics and Laboratory for Space Research, the University of Hong Kong, Pok Fu Lam, Hong Kong SAR, China

*Now at Dipartimento di Chimica e Farmacia, Universita“ di Sassari, I-07100, Sassari, Italy.

$\dagger$ Now at Dipartimento di Fisica e Chimica "E. Segre“”, Universita“ degli Studi di Palermo, via delle Scienze ed. 17, I-90128 Palermo, Italy.

${ }^{\ddagger}$ Also at Institute of Physics, Ecole Polytechnique Federale de Lausanne (EPFL), CH-1015 Lausanne, Switzerland.

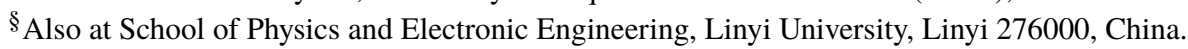

